\title{
KYSTE HYDATIQUE CERVICO MEDIASTINAL A PROPOS D'UN CAS
}

\author{
K. AKKARI, S. CHNITIR, M. BEN MILED, A. MARDASSI, A. SETTHOM, \\ S. HACHICHA ${ }^{* *}$, A. SAKHRI ${ }^{* * *}$, S. BENZARTI, MK. CHEBBI. \\ SERVICE D'ORL ET DE CIRURGIE CERVICO-FACIALE \\ HÔPITAL MILITAIRE DE TUNIS \\ ** SERVICE DE CHIRURGIE CARDIO-VASCULAIRE DE L'HÔPITAL MILITAIRE DE TUNIS \\ ${ }^{* * *}$ SERVICE DE PNEUMOLOGIE DE L'HÔPITAL MILITAIRE DE BIZERTE
}

\begin{abstract}
Le kyste hydatique cervico-médiastinal est extrêmement rare même en pays d'endémie. II se manifeste généralement par une masse cervico-thoracique et il pose un problème délicat de diagnostic.

Les auteurs rapportent le cas d'une femme de 53 ans, qui a consulté pour une masse sus claviculaire gauche évoluant depuis une année. Le bilan radiologique avait conclu au début à un magma d'adénopathies cervico-médiastinales. La patiente a bénéficié, alors, d'une cervicotomie exploratrice avec la découverte en per-opératoire d'un kyste hydatique cervico-médiastinal.
\end{abstract}

Mots clé : Kyste hydatique, localisation cervico-médiastinale, imagerie.

\begin{abstract}
Hydatid cysts of the cervico mediastinal region are very rare, even in countries where echinococcosis is endemic. It presents as a cervical or thoracic mass and shows many diagnosis difficulties.

The authors report a case of a 53 old woman who consulted for a left sus clavicular mass developed from one year. Radiological investigations had concluded, initially, to multiple lymph nodes.

The patient was operated and an hydatid cyst of the cervico mediastinal region was identified.

Key words : Hydatid cyst, cervicomediastinal localization, imagery.
\end{abstract}

\section{INTRODUCTION}

L'hydatidose est une parasitose causée par le développement chez l'homme de la forme larvaire du Taenia Echinococcus (1).

La localisation cervicale est extrêmement rare même en pays d'endémie. Elle se manifeste généralement par une masse cervico thoracique et elle pose un réel problème diagnostique $(2,3)$.

Nous rapportons le cas d'un kyste hydatique cervico médiastinal de découverte per-opératoire.

\section{Notre observation :}

II s'agissait d'une femme âgée de 53 ans hypertendue qui a consulté pour une tuméfaction latéro-cervicale basse et sus claviculaire gauche évoluant depuis une année.

L'examen trouvait une masse jugulo-carotidienne basse et sus claviculaire gauche, ferme faisant $3 \mathrm{~cm}$ de grand axe, fixée au plan profond dont la limite inférieure n'était pas bien perçue, la peau en regard était saine. La nasofibroscopie avait trouvé un bombement régulier de la paroi postérieure du cavum.

L'échographie était en faveur de la présence de deux adénopathies sus claviculaires gauches de 24 et $32 \mathrm{~mm}$ de grand axe, à centre nécrosé.

La radiographie du thorax a montré un élargissement du médiastin supérieur par une opacité cervico-médiastinale supérieure gauche refoulant la trachée (fig. 1).

Une tomodensitométrie a alors été demandée et avait mis en évidence une masse pseudo-tissulaire polylobée prenant le contraste de façon hétérogène intéressant la chaîne ganglionnaire médiastinale antérieure gauche avec extension vers la région cervicale, refoulant le paquet jugulocarotidien et la trachée et s'étendant sur $8 \mathrm{~cm}$, évoquant en premier lieu un magma d'adénopathes (fig. 2 et 3).

Tout le bilan étiologique d'une adénopathie cervicale chronique sus claviculaire gauche était négatif (Bilan infectieux, biopsie du cavum, examen digestif, examen gynécologique).

Une fibroscopie bronchique a été pratiquée et était normale.

La patiente a alors bénéficié d'une cervicotomie exploratrice révélant en per-opératoire un kyste hydatique cervico-médiastinal (figure 4).

Une thoracotomie postéro latérale gauche au niveau du cinquième espace intercostal a alors été réalisée.

L'intervention consistait en une aspiration et stérilisation du contenu du Kyste avec une périkystectomie partielle (le périkyste était épais et adhérant aux corps vertébraux) (Figure 5).

Les suites opératoires étaient simples. Le recul est de un an. 


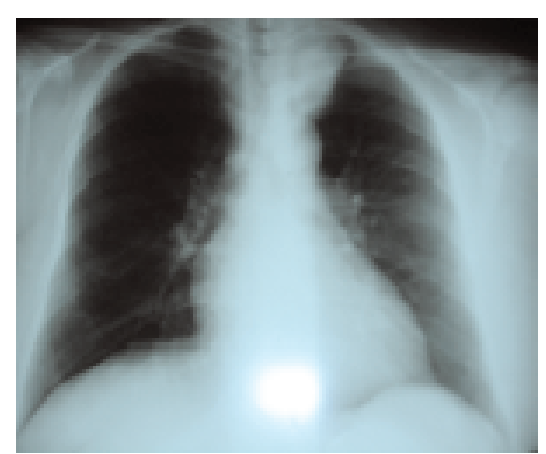

Fig 1 : Radiographie du thorax de face : élargissement du médiastin supérieur gauche.

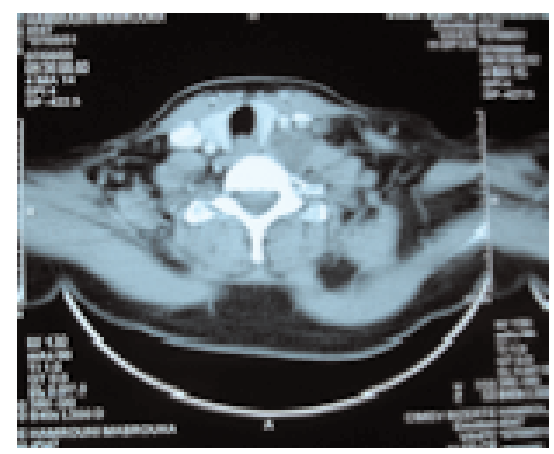

Fig 2 : TDM en coupe axiale montrant la masse au niveau cervical refoulant la trachée et l'axe vasculaire.

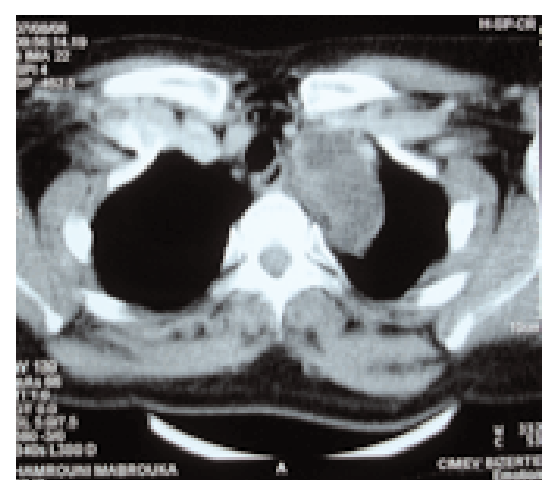

Fig 3 : TDM en coupe axiale montrant la masse au niveau médiastinal postéro supérieur gauche.

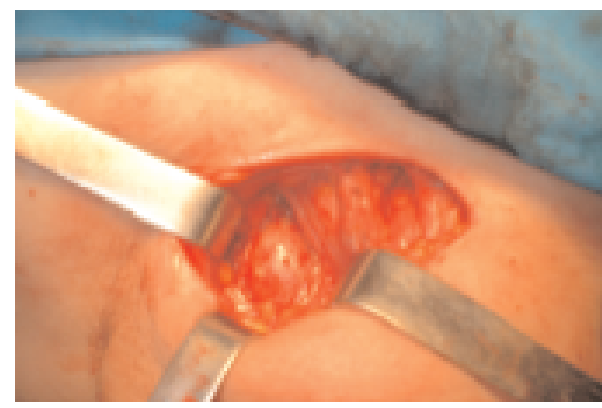

Fig 4 : Vue peropératoire du kyste.

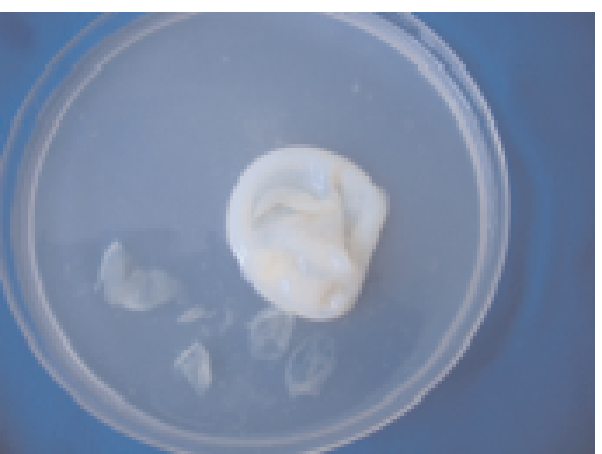

Fig 5 : Membranes hydatiques et vésicules filles.

\section{DISCUSSION}

Le kyste hydatique cervico-médiastinal est très rare et se manifeste par une masse cervicale d'apparition progressive ferme et rénitente. (4)

La localisation médiastinale varie entre 0,1 et $4 \%$ dans la littérature. Elle est de $0,1 \%$ dans les pays occidentaux et de $4 \%$ dans les pays d'endémie (3,9\% en Tunisie). $(2,5)$ Deux mécanismes peuvent être en cause : le parasite après avoir franchi le filtre hépatique et pulmonaire pénètre dans la circulation sanguine systémique et se fixe dans le médiastin, le second mécanisme est celui d'un cheminement par les voies chylifères et lymphatiques (2). Toutes les localisations médiastinales sont possibles avec une prédilection pour le médiastin postérieur $(2,5)$.

L'atteinte des tissus mous est rare. Le kyste hydatique atteint généralement les muscles du cou, du tronc et de la racine des membres (4).

La symptomatologie clinique est polymorphe et se manifeste classiquement par une masse rénitente indolore et lentement progressive $(2,4,6)$.

Les signes d'irritation ou de compression des organes de voisinage peuvent s'y associer $(2,5,6,7)$. Le kyste hydatique du médiastin postérieur peut entraîner des douleurs et des signes neurologiques avec possibilité d'érosion des côtes et des vertèbres (2). Notre patiente avait un kyste du médiastin postérieur avec des signes de compression neurologique à type de douleurs du membre supérieur gauche.

Rarement le kyste hydatique peut se manifester par des signes évoquant une fissuration ou une rupture.

L'imagerie joue un rôle essentiel dans le diagnostic positif et le bilan d'extension des lésions.

La radiographie standard montre un élargissement du médiastin par une masse de tonalité hydrique $(3,4,5)$. Les calcifications de la paroi s'observent au cours du kyste hydatique primitif du médiastin. Elles sont rares mais représentent un argument diagnostique non négligeable (2).

L'échographie est un examen très performant qui permet de reconnaître le caractère liquidien de la masse et la 
paroi fine du kyste $(2,4,5)$. Elle objective des aspects comparables à ceux des autres localisations viscérales, en particulier hépatiques répondant à la classification de Gharbi (4).

La TDM montre une masse de densité liquidienne bien limitée non modifiée par le produit contraste, et dont les limites sont nettes. Elle est généralement uni rarement multi vésiculaire. Dans le cas de notre patiente il s'agissait d'une opacité multi vésiculaire.

L'IRM a un intérêt anatomique. Le kyste hydatique apparaît en hypo-signal sur les séquences pondérée T1 et en hyper-signal sur les séquences pondérées T2. La paroi du kyste apparaît sur les séquences T2 sous forme de deux couches de signal différent : la couche périphérique (le périkyste) est en hypersignal prenant le produit de contraste, et la couche interne est hypointense ne prenant pas le produit de contraste (4). L'IRM peut aussi être utile au diagnostic en montrant les vésicules filles à l'intérieur du kyste.

Le traitement est toujours chirurgical et permet la kystectomie et la périkystectomie. La périkystectomie totale emportant le kyste fermé représente la méthode de choix. La périkystectomie subtotale est réservée aux cas où le kyste adhère aux axes vasculo-nerveux. Notre patiente avait bénéficié d'une périkystectomie partielle à cause d'une adhérence du kyste aux corps vertébraux.

Le résultat est bon dans la plupart des séries rapportées.

\section{CONCLUSION}

Le kyste hydatique cervico-médiastinal est extrêmement rare. L'imagerie doit normalement permettre le diagnostic positif, ainsi que le bilan d'extension aux organes de voisinages. Son traitement est chirurgical et l'évolution postthérapeutique est souvent bonne.

\section{REFERENCES}

1) Audouin J, Delevoux M, et al. A propos d'un cas de kyste hydatique du médiastin en république de Niger. Méd Trop $1986 ; 46: 401-4$.

2) El Abaissi Skalli A, El Amraoui F, Chikhaoui N. Kyste hydatique du médiastin à propos de deux cas. J Radiol 2000; 81: 154 - 157.

3) Ben Miled-M'rad K, Bouricha A, Hantous S. Apport de l'imagerie au diagnostic du kyste hydatique de la paroi thoracique. J Radiol 2003; 84: 143 - 146. 4) Beji M, Ben Massoud M, Louzir B. Localisation cervico-thoracique d'un kyste hydatique. J Radiol 2004; 85: 135 - 137.
5) Les kystes hydatiques du thorax : rapport de la société Tunisienne de chirurgie, Avril 1991

6) Aloui Yazidi A, Sibaï H, Kafih A et al. Syndrome de Pancoast Tobias d'origine hydatique. Rev Mal Respir $1995 ; 12: 49-52$

7) Abi F, El Fares F, et al. Les localisations inhabituelles du kyste hydatique. À propos de 40 cas. J chir $1989 ; 126: 307-12$. 